\title{
Crítica desde la filosofía analítica de Donald Davidson a una ontología de lo humano basada en criterios de atribución de racionalidad: el caso de George Floyd ${ }^{*}$
}

\section{Criticism from Donald Davidson's analytical philosophy to an ontology of the human based on attribution criteria of rationality: the case of George Floyd}

Jose Jeyson Fernando Espejo Moya*

\section{Resumen}

La presente reflexión tiene por objeto establecer una crítica a una ontología de lo humano basada en criterios de atribución de racionalidad, a partir de la propuesta epistemológica y ética presente en el pensamiento filosófico del profesor Donald Davidson. Para tal fin se tomará como objeto de análisis el caso de la muerte violenta de George Floyd bajo custodia policial en mayo de 2020 y cómo se incurrió en errores de atribución de racionalidad en su caso. A modo de conclusión, se sostiene que fundamentar una ontología

Artículo de Reflexión.

** Licenciado en filosofía, pensamiento político y económico de la Universidad Santo Tomás, Estudiante de Maestría en filosofía contemporánea de la Universidad de San Buenaventura, sede Bogotá. Contacto: jfespejom@academia.usbbog.edu.co | https://orcid.org/0000-00016607-3091 
de lo humano resulta en fracaso porque el criterio, o los criterios para atribuir racionalidad desde los que se ha partido no obedecen a juicios valorativos objetivos, sino a prejuicios que no soportan un análisis lógico riguroso.

\section{Palabras clave:}

Atribución de racionalidad, objetividad de los valores, ética práctica, ontología de lo humano, George Floyd.

\section{Abstract}

The following reflection aims to establish a critique to a human's ontology based on criteria of attribution of rationality, on the basis of the epistemological and ethical proposal present in the philosophical thought of Professor Donald Davidson. For this purpose, it will be taken the case of the violent death of George Floyd in police custody in May 2020 and how errors of attribution of rationality were incurred in his case. As a conclusion, it is argued that establishing an ontology of the human results in a failure because the criterion, or the criteria from which rationality is attributed, do not obey to objective evaluative judgments, but to prejudices that do not support a rigorous, logical analysis.

\section{Keywords:}

Attribution of rationality, objectivity of values, practical ethics, ontology of the human.

"No importa cómo lo digamos, sigue habiendo esa diferencia, por lo que respecta a la racionalidad, entre los bebés y los caracoles, por una parte, y las personas adultas normales por otra”.

(Davidson, 1997). 


\section{Introducción}

La tesis que se trata de defender en el presente ensayo sostiene que la propuesta analítica de Donald Davidson es un insumo para demostrar por qué se incurre en un error al tratar de fundamentar una ontología de lo humano con base en criterios de atribución de racionalidad. La argumentación de la cual se hará uso operará en tres etapas. En primer lugar, se indicará por qué resulta problemática una ontología de lo humano basada en criterios de atribución de racionalidad, y para ello se mostrará en qué consiste el concepto de la tiranía de lo racional. Posteriormente, se presentará la teoría de la objetividad de los valores $\left(O V^{I}\right)$ de Davidson y cómo esta permite dar luces sobre el problema enunciado, tomando como recurso el caso concreto de atribución de racionalidad adelantado sobre George Floyd por cuenta de sus victimarios para demostrar por qué se ha incurrido en errores al respecto. Acto seguido, se indicarán las razones por las cuales el criterio de atribución de racionalidad, propuesto por Davidson en Animales racionales $\left(R A^{2}\right)$, es objetivo y permite abordar el problema desde una óptica distinta. Para finalizar, se delinearán las conclusiones a las cuales nos lleva el recorrido argumentativo y en qué posición queda la tesis propuesta.

\section{La tiranía de lo racional y sus implicaciones ontológicas}

Si bien desde la Antigüedad se ha planteado que la racionalidad es un rasgo distintivamente humano, fue con el inicio de la Modernidad que esta idea tomó una fuerza tal que desplazó cualquier otra posibilidad de definir dicho estatuto ontológico: el cogito se estableció como el determinante para definir quién era humano y quién no. Fue este 'principio' el que acompañó y, en muchos casos, el que impulsó los distintos procesos de colonización europea alrededor

1 Abreviatura usada en correspondencia al título original del artículo en inglés The Objectivity of Values (1997b).

2 En correspondencia al original en inglés Rational Animals (1997a). 
del planeta, iniciados con los viajes de Colón, y que han configurado el estado actual de las cosas en términos de un mundo globalizado que, por lo menos en una buena parte, sigue teniendo a Europa ${ }^{3}$ como punto de referencia central.

Dicho proceder de los acontecimientos históricos resultaría inocente de no ser porque, en medio del proceso de expansión de la racionalidad europea, han tenido lugar numerosas prácticas de dominación y exterminio hacia otros pueblos e, incluso, hacia civilizaciones enteras, por cuenta -en parte- de una presunta superioridad ontológica, de la cual gozara el europeo por asumirse más racional en comparación con los individuos sujetos de colonización. Es así como 11 millones de esclavos subsaharianos arribaron a América entre los siglos XVI y XVIII (Hobsbawm, 2001, p. 48) y padecieron sufrimientos indecibles porque, según la visión de sus negreros, al no poseer alma, no se trataba de seres humanos y se podía disponer de ellos como se haría con cualquier otro objeto de propiedad. Un destino similar cobijó -solo en el Caribe- a los 10 millones de nativos indígenas que murieron a manos de no más de diez mil españoles (Reséndez, 2016, pp. 16-17), quienes determinaron no atribuir humanidad a los indios y contribuir así, en conjunto, a lo que más adelante Galeano catalogara como el otrocidio de las Américas.

Si se decidiera seguir el rastro al devenir de las implicaciones de esta tiranía de lo racional, nos encontraríamos en la historia con múltiples acontecimientos en los que al decidir atribuir humanidad por cuenta de un supuesto de no-racionalidad arbitrario, ejercido en contra del otro, se decidió sobre la vida de tantas y tantos y sobre sus posibilidades de existencia. Ahora bien, se enfatizará en la mentada

3 Debe entenderse el concepto no solo como la circunscripción geográfica, sino como la herencia de un tipo de racionalidad que ha dominado desde que la Modernidad fue puesta en marcha y que cobija, por ejemplo, las lógicas que han movido a los Estados Unidos en los últimos siglos. 
arbitrariedad de la atribución de racionalidad-humanidad porque es probable que allí resida el núcleo de la cuestión que constituye el interés central del presente análisis. La historia indica que los colonizadores europeos, que en adelante pasaremos a denominar bajo la figura del "atribuyente", definieron que indígenas y negros no eran humanos porque carecían de alma o cualquier otro atributo que -desde su visión- era muestra irrefutable de racionalidad. Es evidente que la decisión se tomaba con base en una percepción de que el propio sistema cultural europeo era el punto cero (y exclusivo) de enunciación: el "atribuyente" determinaba lo racional sin poner en cuestión la legitimidad de dicha determinación. Se daba por sentado que se estaba en posesión de la verdad y el respaldo era una mayor eficiencia para ejercer violencia contra del indígena o el negro, es decir, del "despojado". Pero ¿́cuán objetivo realmente es ese criterio de atribución de racionalidad? Si hipotéticamente se retirara la violencia de la ecuación, ¿̇on qué elementos contaría el "atribuyente" para legitimar su decisión de atribución de racionalidad-humanidad sobre un potencial "despojado"? ¿Se trata, de hecho, de un acto racional atribuir (o no) racionalidad a otro ser a partir de criterios arbitrarios $\mathrm{y}$, aparentemente subjetivos, convenientemente ordenados al servicio de una empresa de dominación sobre el otro?

Casi que sin darse cuenta, la conquista europea de ultramar estableció con mayor o menor éxito -eso depende del punto desde dónde se observe- un sistema ontológico con terribles implicaciones de orden práctico-existencial para las y los desafortunados que quedaron fuera de su amparo. A fuerza de prácticas irracionales, esta forma de la racionalidad europea determinó quién podía ser humano e hizo uso a discreción de quienes quedaron en la acera de los "despojados" que perdieron su humanidad. 


\section{La teoría de la objetividad de los valores: caso George Floyd}

Para demostrar por qué se considera que dichas prácticas son irracionales, que no obedecen a criterios de objetividad y que, por tanto, son arbitrarias, se hará uso de la teoría de la objetividad de los valores de Donald Davidson. Perteneciente a la vertiente de la filosofía analítica, el profesor estadounidense está convencido de que un análisis riguroso del lenguaje permite dar cuenta, de un modo más preciso, de objetos de estudio que otros enfoques de la filosofía han abordado de manera más bien poco sistemática. En este caso, la cuestión ética pasa a ser estudiada a través de los enunciados que se pueden emitir alrededor de un acto o de un objeto. Davidson sostiene, entonces, que "los valores están enraizados en las cosas" (1997b, p. 52), y su argumento es que las mismas propiedades descriptivas de las cosas o de los hechos 'causan' las mismas actitudes/creencias en diferentes observadores, lo que, en otras palabras, quiere decir que los valores son objetivos según estándares interpersonales y, en consecuencia, no responden a determinaciones subjetivas. La evidencia de las actitudes o las creencias están presentes en las proposiciones emitidas por los sujetos y son estas a las cuales deben ir dirigidos los intereses analíticos. Es así que, dentro de ese marco de estudio definido, el autor propone, como ya se ha visto: 1. los valores son objetivos porque residen en las 'cosas' (o en los hechos); por tanto, 2. los juicios valorativos son objetivos (es decir, verdaderos o falsos) en la medida -y solo en ese caso- en la que su contenido es determinado por los hechos; $y$, en consecuencia, 3. el valor de verdad de tales juicios se determina a partir de la correspondencia entre los hechos y el contenido de la proposición juzgada (Davidson, 1997b, p. 45).

Ahora bien, ¿́cómo vincular estos postulados de la objetividad de los valores con el problema presentado anteriormente? La atribución arbitraria de racionalidad por parte de los colonizadores europeos y sus consecuencias no poseerían mayor atractivo, más 
allá de una eventual reivindicación de orden histórico - sin que con ello se niegue su importancia y su necesidad-, sino fuera porque la colonización tuvo tal éxito que actualmente en las prácticas sociales cotidianas se decide quién es más o menos humano, siguiendo los mismos patrones de atribución de racionalidad que ya se han puesto en cuestión. La diferencia está en que ya no hay una distinción tan marcada entre una mentalidad europea y una otra. En ese sentido, la herencia de Europa configuró el mundo occidental y, en consecuencia, cualquiera está en riesgo de actuar como "atribuyente" $\mathrm{y}$, al mismo tiempo, pareciera que todos se hallan indefensos ante un potencial despojo de la propia humanidad por cuenta de que sean asumidos lo suficientemente poco racionales para desmerecer el estatuto ontológico necesario. En ese sentido, pareciera que todos en Occidente han heredado el vicio de atribución arbitraria de racionalidad de los colonizadores y, por esa razón, todos poseen algo de europeos en su bagaje cultural ${ }^{4}$; a eso se hace referencia cuando se afirma que la colonización tuvo éxito. La colonización instauró una definición de normalidad más o menos clara y determinó que quienes, de una u otra manera, excedan sus límites son potenciales "despojados" de humanidad. En consecuencia, hoy en día no solo se corre el riesgo de ser "despojado" por cuestiones raciales, sino por cualquier tipo de divergencia que no se alinee con el canon de normalidad impuesto. En suma, y bajo el riesgo de incurrir en reiteraciones, las prácticas arbitrarias de atribución de racionalidad presentes en los colonizadores europeos son las mismas de las cuales se hace uso actualmente cuando la singularidad se enfrenta al otro. Por esa razón, se procederá a aplicar los postulados de la objetividad

4 Con estas afirmaciones no se pretende defender una suerte de lógica maniquea, ni estigmatizar a los pueblos europeos, tampoco negar los aportes significativos y determinantes que ha dado la racionalidad europea para la configuración del mundo contemporáneo, ni mucho menos caer en simplificaciones del tipo: los europeos albergan aún ideas de dominación sobre otros pueblos e insisten en negar su humanidad. La crítica va dirigida exclusivamente a una suerte de eurocentrismo mental cerrado que le ha sido legado a la contemporaneidad occidental y que debe ser puesto en cuestión porque opera de la misma forma en la que lo hacía 500 años atrás, sabiendo los riesgos que ello comporta, solo que ahora todos pueden asumir tanto el rol de "atribuyente" como el de "despojado". 
de los valores de Davidson a una situación de actualidad para dar cuenta de por qué las prácticas denunciadas en los colonizadores europeos eran arbitrarias, y, a su vez, se tratará de hacer evidente que, por extensión, dichas prácticas son análogas a las que se viven hoy en día y que, en consecuencia, el problema persiste.

A inicios de junio de 2020 el movimiento Black Lives Matter cobró un fuerte impulso en su lucha contra el racismo a raíz de la muerte de George Floyd bajo custodia policial en la ciudad de Minneapolis (Minnesota). Las impactantes imágenes del policía imprimiendo el peso de su cuerpo con su rodilla en el cuello de Floyd mientras que este con voz agónica repetía 'I can't breath' fueron noticia mundial. De acuerdo con los postulados de Davidson, podrían darse dos tipos de proposiciones antagónicas sobre el mismo hecho, que se denominará $\mathrm{X}$ por cuestiones meramente prácticas.

Podría darse, por una parte, que ante el hecho un observador $\mathrm{O}^{1}$ emitiera un juicio valorativo del tipo «X es indignante». En primer lugar se debería analizar si $\mathrm{X}$ es objetivamente indignante; es decir, $\mathrm{X}$ será objetivamente indignante siempre y cuando las mismas propiedades descriptivas de $\mathrm{X}$ consideradas por distintos observadores generen la misma actitud: la indignación. Siendo así, X es indignante; lo que en otros términos implicaría que en $\mathrm{X}$ reside la indignación y, por tanto, dicho valor es objetivo. En segundo lugar, se debe determinar si la proposición «X es indignante» es objetiva o no. Davidson dice que la objetividad de los juicios valorativos depende de que su contenido esté determinado por los hechos, tal y como sucede en este caso; por tanto, la proposición es objetiva. Por último, hay que determinar el valor de verdad del juicio valorativo con base en la correspondencia entre los hechos y el contenido de la proposición. En este caso, dado que hay coherencia entre la objetividad del valor atribuido al hecho y la proposición que da cuenta de ello, es posible afirmar que el juicio valorativo es verdadero. Por otra parte, podría darse que ante el hecho un observador $\mathrm{O}^{2}$ 
emitiera un juicio valorativo del tipo «X es justo» $\mathrm{y}$ que tratara de argumentar que tanto el hecho es objetivamente justo como que su proposición es objetiva. $\mathrm{O}^{2}$ estaría llevado a pensar que «X es justo» por cuenta de que Floyd estaba cometiendo un delito. En ese caso $\mathrm{O}^{2}$ no está considerando las mismas propiedades descriptivas que $\mathrm{O}^{1}$; es decir, está yendo más allá del hecho en cuestión y, por tal razón, ni hay evidencia objetiva de justicia en $\mathrm{X}$, ni la proposición de $\mathrm{O}^{2}$ es objetiva. En consecuencia, no es posible determinar valor de verdad en el juicio valorativo de $\mathrm{O}^{2}$ por cuenta de que no es objetivo de acuerdo con la lógica derivada de la propuesta de Davidson.

Un aspecto importante de los postulados del autor al respecto de la objetividad del contenido de un juicio valorativo es que esta pasa por un ejercicio de interpretación sobre las proposiciones de otra persona. Por tal razón, Davidson (1997b) sugiere que

un intérprete no puede esperar determinar los contenidos de los deseos de una persona sin determinar también lo que la persona cree; y no hay manera de determinar los contenidos de ninguna de esas actitudes de modo suficientemente detallado sin commnicación lingüistica, la cual requiere la interpretación del habla de la persona. (p. 49, cursiva fuera de texto)

En ese orden, solo en situaciones comunicativas es posible corroborar los contenidos de las actitudes de otra persona. No se trata, por tanto, de un ejercicio a priori en el que, asumiendo que se conoce el lenguaje de la otra persona, puede hacerse una idea efectiva de los contenidos de sus proposiciones o, peor aún, de si da cuenta o no siquiera de proposición alguna.

\section{Repensar la atribución de racionalidad desde la filosofía analítica de Davidson}

Una vez visto cómo opera la teoría davidsoniana con relación a la objetividad de los valores y de las proposiciones de los juicios valorativos, se intentará arrojar algunas luces más propiamente sobre 
el tema de interés de la presente reflexión: la atribución arbitraria de racionalidad y, en consecuencia, de humanidad por parte de una persona hacia otra.

Para ello es necesario volver al caso de Floyd. Si bien su muerte fue real, es posible considerar -a partir de un ejercicio meramente especulativo-que las condiciones que llevaron a que el policía actuara como actuó pertenecen más bien al plano lingüístico. Asúmase, no sin malicia, que el oficial de policía Derek Chauvin alberga ciertas ideas supremacistas de una manera más o menos discreta. Asúmase que se da el caso de que el policía emitió los siguientes juicios valorativos con relación a Floyd y que lo llevaron a adelantar las acciones ya conocidas:

1. George no es blanco;

2. George está cometiendo un delito;

3. George no es un ser (completamente) racional;

4. George no es (completamente) humano;

5. George no merece un trato (completamente) humano.

Aplicando la teoría de Davidson es posible decir que en la proposición 1 se hace evidente un prejuicio que opera en la mente de Chauvin, que lo lleva a considerar que se encuentra en una posición de superioridad frente a Floyd por una cuestión racial que, combinada con su posición de agente de la ley y lo enunciado en la proposición 2, lo llevó rápidamente a concluir que las proposiciones 3,4 y 5 eran acertadas. De todos modos, es evidente que la proposición l no debería haber jugado un rol determinante en la acción de Chauvin; sin embargo, pareciera que sí lo fue. De hecho, pareciera que en el caso de que se tratara de un supremacista, se podría pasar de la proposición 1 a la 3 sin mayores dificultades, por cuenta de las creencias que conllevan a considerar que hay razas superiores a otras y que su lugar de enunciación es el de "atribuyente". Siguiendo 
con Davidson, resultaría natural admitir 1 y 2, pero el problema residiría en 3. El problema es la atribución de un menor grado de racionalidad por cuenta de un criterio no objetivo (asumir que alguien no es racional porque es negro, o porque comete un delito, o por ambas razones combinadas). Ahora bien, no habría mayor problema si este tipo de consideraciones hubiesen permanecido en el plano de las creencias solamente; la dificultad mayor está en que estas consideraciones ontológicas llevaron a que Floyd terminara muerto, independientemente de que las intenciones de Chauvin no necesariamente hayan sido que el desenlace fuera tal. El discurso del abolicionismo cuenta con evidencias contundentes para demostrar que en los casos en los que los delincuentes son blancos reciben mejores tratos que cuando se trata de personas negras, lo que lleva a concluir que hay un prejuicio racial que determina el grado de maltrato que puede recibir un delincuente, que, por regla general, desemboca en casos de exceso en el uso de la fuerza por parte de la fuerza pública ${ }^{5}$.

Así como con el caso de Floyd, ha de asumirse que las circunstancias de atribución de racionalidad por parte de los colonizadores a los negros y a los indígenas cinco siglos atrás no fueron distintas: ya fuera porque se consideraba que no tenían alma, o porque desde la visión del conquistador su lenguaje no superaba los gruñidos de cualquier otra bestia, se negaban la racionalidad y la humanidad a estas poblaciones con base en criterios subjetivos de atribución y, por tanto, arbitrarios. En todos los casos se ha fallado en atribuir racionalidad por cuenta de la existencia de prejuicios en las valoraciones hacia el otro y, además, porque no se dio una comunicación lingüística efectiva para corroborar los contenidos

5 Para el caso colombiano consultar las siguientes investigaciones: Lam, Y. y Ávila Ceballos, C. (2013). Orden público y perfiles raciales. Experiencias de afrocolombianos con la policía en Cali. Centro de Estudios de Derecho, Justicia y Sociedad, Dejusticia. Asimismo, Gil Hernández, F. (2010). Vivir en un mundo de 'blancos'. Experiencias, reflexiones y representaciones de 'raza' y clase de personas negras de sectores medios en Bogotá D.C. [Universidad Nacional de Colombia]. http://www.bdigital.unal.edu.co/3135/1/478264.2010.pdf 
de las actitudes de la contraparte. Davidson añadiría que, además, en estos casos ha habido una falla en la política de acomodación racional o principio de caridad desde el cual "la interpretación [de las proposiciones de los demás] implica ver las vidas mentales de otros y la nuestra como absolutamente coherentes y correctas" (1997b, p. 50). Por supuesto, en el caso de los conquistadores, y probablemente en el del oficial de policía, no había una disposición empática hacia el otro. En el primer caso, más que en el segundo, no habría error al afirmar que la negación de racionalidad era deliberada y conveniente y, por tal razón, era imposible asumir como coherente la vida mental del otro. Por el contrario, era 'necesario' determinar que en el otro no había tal vida mental. En el segundo caso no es posible hacer la misma afirmación de manera tan categórica; sencillamente, junto con Davidson, ha de asumirse que hubo un fallo en la interpretación de la vida mental del otro como absolutamente coherente, junto con un fallo en la atribución misma de racionalidad.

Ahora bien, para tratar de responder al problema, el mismo Davidson cuenta con una teoría objetiva para determinar quién es racional y quién no que podría - por lo menos teóricamentefraguar una solución en la que la atribución no depende de criterios arbitrarios. En $R A$ Davidson (1997a) propone que solo poseen racionalidad aquellos animales que sean capaces de dar cuenta de actitudes proposicionales (AP), como creencias, deseos, intenciones o valores (p. 144), independientemente de cuán lógicas o coherentes sean. Dichas AP se sustentan desde el holismo de lo mental; es decir, un sujeto no puede tener una creencia sin que implique poseer otras y, por esa misma línea, poseer deseos, intenciones y valores. Se trata de una compleja red de relaciones en la que las AP dependen unas de otras para establecerse como tales. Por supuesto, la evidencia de que un animal da cuenta de AP se da en sus enunciados lingüísticos, lo que lleva a inferir que es el lenguaje la condición de posibilidad para evidenciar y atribuir racionalidad a un animal. La intención de Davidson, naturalmente, no es la de establecer un sistema ontológico 
a partir de la atribución de racionalidad; él simplemente se esfuerza en enunciar las condiciones a partir de las cuales un ser puede considerarse racional o no. De todos modos, al atribuir racionalidad y, eventualmente, humanidad por esta línea se dejan a muchas menos personas por fuera que desde la otra situación ontológica ${ }^{6}$.

Sería posible, a partir de esta nueva teoría, hacer una ulterior revisión del caso de Floyd y, por extensión, de lo sucedido en los procesos de colonización. En un caso hipotético Chauvin podría emitir los siguientes juicios valorativos:

1. George poseía actitudes proposicionales y actitudes valorativas;

2. George era un ser racional;

3. solo los humanos son racionales;

4. George era humano;

5. George merecía un trato digno para su humanidad.

De 1 se puede decir que es objetivo por cuenta de que Floyd pretendía pagar con un billete falso y dicha pretensión es evidencia de que poseía AP; por tanto, 2 también es objetivo. De ello se desglosan fácilmente 4 y 5 y no es arriesgado asegurar que las 5 proposiciones son verdaderas.

Si los sucesos se hubiesen configurado de una manera similar, muy probablemente Floyd estaría preso, pero vivo y Chauvin estaría libre y conservaría su trabajo. Esto, claro, si hubiese habido una situación comunicativa favorable y si Chauvin hubiese aplicado el principio de caridad hacia Floyd. Otorgue el lector, por favor, implicaciones similares a partir de la misma argumentación para el asunto

6 Davidson en $R A$ está de acuerdo en que los bebés y los discapacitados mentales no son seres racionales, pero nunca asume que no son humanos. 
de los colonizadores frente a los negros y los indígenas, salvando cuidadosamente las respectivas distancias.

Por último, Davidson diría que en los casos que fueron objeto del presente análisis ni siquiera se trataba necesariamente de una cuestión ontológica, sino que el problema obedece más bien a cuestiones epistemológicas con unas enormes implicaciones éticas. Epistemológicas porque se trata de determinar la objetividad del contenido de unas proposiciones y, en consecuencia, de los valores que se le atribuyen a los otros y a sus actos a través de los juicios valorativos del atribuyente. En ello están en juego las condiciones de posibilidad del conocimiento que cada quien puede tener de las AP del otro. Las implicaciones éticas ya han sido ampliamente expuestas y no se insistirá en ellas.

\section{Conclusión}

Para finalizar, Davidson estaría de acuerdo en que los intentos de fundamentar una ontología de lo humano han fracasado porque el criterio, o los criterios para atribuir racionalidad desde los que han partido no obedecen a juicios valorativos objetivos, sino a prejuicios que no soportan un análisis lógico riguroso, evidencia, estos últimos, de una falla en el principio de caridad y de que han sido emitidos al margen de situaciones comunicativas que permitan dar cuenta de los contenidos de las AP del otro. Es más, insistir en fundamentar una ontología de lo humano a partir de la atribución de racionalidad, desde los postulados de Davidson, sería una necedad.

De todas maneras, se ha hecho -y se sigue haciendo en nuestras prácticas cotidianas- y ello supone un error que ha servido de andamiaje para justificar prácticas discriminatorias y de dominación; por tanto, no es inocente y debe corregirse para que menos gente muera porque se la considere menos humana que otra. 


\section{Referencias}

Davidson, D. (1997a). Animales racionales. En Subjetivo, intersubjetivo, objetivo (pp. 141155). Cátedra.

Davidson, D. (1997b). La objetividad de los valores (G. Cobo, Trad.). Estudios de filosofía, 3, 43-52.

Hobsbawm, E. (2001). Industria e imperio. Historia de Gran Bretaña desde 1750 hasta nuestros días (G. Pontón, Trad.). Crítica.

Reséndez, A. (2016). Caribbean Debacle. En The Other Slavery: The Uncovered Story of Indian Enslavement in America. Houghton Mifflin Harcourt. 